\title{
Integrating Catalytic Processes and Modern Electrolyte Concepts into Electrosynthesis
}

\author{
Robert Francke*
}

\begin{abstract}
Electrosynthesis is frequently presented as an intrinsically sustainable, safe and efficient method. While this is indeed often the case, this assessment cannot be easily generalized, as a number of challenges need to be addressed on the way to more efficient and truly sustainable processes. These challenges comprise the necessity for employing large amounts of supporting electrolyte additives along with the concomitant separation and waste issues. A further problem is the kinetic inhibition of the heterogeneous electron exchange, which in many instances leads to a decreased selectivity and an increased energy consumption ('overpotential'). Another challenge is the apparent restriction of electrosynthesis to redox reactions, which seems to exclude important redox-neutral processes such as rearrangements, cycloadditions and substitutions from the scope of applications. Herein, catalytic approaches and electrolyte concepts are presented, which can help to overcome the abovementioned issues. For illustration of the principles, examples from our recent research activities are used.
\end{abstract}

Keywords: Electrocatalysis · Electrosynthesis · Hypervalent iodine · Molecular rearrangement · Polyelectrolyte

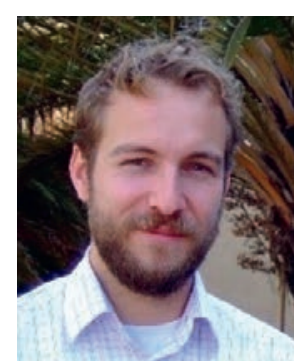

Robert Francke studied chemistry at Bonn University (Germany) and Alicante University (Spain). He received his diploma degree (equivalent to a MSc degree) in 2008 from Bonn University, where he subsequently started working on his dissertation in the group of Prof. S. R. Waldvogel. After relocation of the Waldvogel Group, he finished his dissertation on fluorinated electrolytes for electrochemical energy storage devices at Mainz University (Germany) in 2012. Equipped with a Feodor Lynen Fellowship (Alexander von Humboldt Foundation), he then joined the group of Prof. R. D. Little at the University of California, Santa Barbara, where he started his research in the field of organic electrosynthesis. In 2014, he returned to Germany to start his independent career at Rostock University as a Liebig Fellow (Fonds der Chemischen Industrie). His current research takes place at the interface between organic chemistry, electrochemistry and technical chemistry and is focused on the development of efficient and sustainable syntheses involving various reactor concepts and separation processes.

\section{Introduction}

Compared to conventional methods for the oxidation and reduction of chemical compounds, electrosynthesis offers several economic, ecological and practical advantages. ${ }^{[1-3]}$ On the one hand, the use of electricity instead of redox reagents reduces the waste generation of a process and can thereby help to improve the sustainability and cost-efficiency. On the other hand, the electrode potential as the driving force of the reaction is continuously variable and can be precisely adjusted to the redox potential of a specific chemical compound in solution. Furthermore, electrosynthesis is typically carried out under mild conditions (room temperature and atmospheric pressure) and can be considered as inherently safe (controlled in situ generation of highly reactive intermediates or reagents).
All these advantages were recognized long ago and have been used for a variety of laboratory-scale applications, including the synthesis of complex organic scaffolds ${ }^{[4]}$ or the electrochemical valorization of $\mathrm{CO}_{2} \cdot{ }^{[5]}$ However, in contrast to the technically mature chlor-alkali and metal deposition processes, ${ }^{[6]}$ the electrochemical conversion of carbon-based compounds such as $\mathrm{CO}_{2}$ and organic molecules has not yet been established on a broad basis in industry. ${ }^{[7-11]}$ Despite all the advantages of the method discussed above, the possibilities are therefore still far from being exhausted. Apart from economic and engineering issues, further challenges along with the possible solutions have been identified:

1) Kinetic inhibition of the heterogeneous electron transfer. This step is often associated with a high activation barrier, which leads to a low selectivity with respect to the desired product and increased energy consumption ('overpotential'). In such cases, homogeneous electrocatalysts ('redox mediators') can help to improve the kinetics of the process ('indirect electrosynthesis', see Section 2). ${ }^{[12]}$ A part of our current work therefore focuses on the development of efficient catalytic systems with regard to both the utilization of $\mathrm{CO}_{2}$ and the conversion of organic compounds.

2) Restriction of the scope to redox reactions. Since the electrolysis of chemical compounds inevitably leads to oxidation or reduction processes, it appears that the scope of the method is limited to redox reactions. However, a few examples from the literature which have received little attention so far show that the method can in principle also be applied to redox-neutral reactions such as isomerizations, rearrangements, substitutions and cycloadditions. ${ }^{[13]}$ Here, the use of sub-stoichiometric charge quantities leads under certain circumstances to the catalysis of these reactions. The approach is also referred to as 'electrochemical catalysis' and constitutes a part of our research program (see Section 3).

3) Necessity for supporting electrolytes. The need to use large quantities of salt additives represents a further challenge. Once 
the reaction is complete, the salt must be isolated from the reaction mixture in an additional separation step. Since the supporting electrolyte additive is both a potential source of waste and a significant cost factor, its recycling in industrial applications is highly desirable. ${ }^{[8]} \mathrm{A}$ part of our research is therefore devoted to the development of strategies for the facilitation of separation and recycling of salt additives and electrocatalysts.

The intent of this review is to discuss these three challenges along with possible solutions using recent examples from our group. Readers interested in comprehensive reviews on the outlined topics are referred to refs. [5], [12] and [13].

\section{Indirect Electrosynthesis}

\subsection{Fundamentals}

The electrochemical conversion of a compound consists of two different types of elementary reactions (electrochemical and chemical steps) and is exemplified in the following for an anodic oxidation. In the case of a direct conversion at the anode, the reaction sequence is initiated by an electron exchange between the polarized electrode and the substrate A dissolved in the electrolyte (see Fig. 1, left). This initial electrochemical step leads to the formation of a reactive intermediate $B$ (typically a radical ion), which further reacts in one or several chemical reactions. In general, a product is thus obtained after a more or less complicated sequence of electrochemical and chemical steps (EC, ECEC, EECC, etc.). In most cases, however, it is precisely the initial electron transfer that represents the bottleneck of the sequence, which is explained with a high activation barrier and the unfavorable energetic position of the reactive intermediate. In electrochemical terms, the activation barrier is expressed as the overpotential $(\eta)$, which has to be applied in addition to the thermodynamic redox potential of the A/B couple to induce the electron exchange. Since high overpotentials lead to an increased energy consumption and a reduced product selectivity, the use of electrocatalysts is often necessary. While heterogeneous electrocatalysts (catalytically active electrodes) are frequently used for the conversion of small molecules, ${ }^{[14]}$ homogeneous electrocatalysts (often referred to as 'redox mediators') are more common for the conversion of organic compounds. ${ }^{[12]}$ In the latter case, a catalyst couple Med I Med $_{\text {red }}$ is interposed between the electrode and the substrate in solution (see Scheme 1, middle) and lowers the reaction barrier via formation of a transient adduct between the active form of the catalyst $\left(\mathrm{Med}_{\mathrm{ox}}\right)$ and A.[15]

In order to allow for a continuous regeneration of the active form of the catalyst in a one-pot process, the catalyst potential must be less positive than the oxidation potential of $\mathrm{A}$ (in a cathodic process: less negative than the reduction potential of A), since otherwise the substrate would be directly converted at the electrode. In the realm of organic electrochemistry, this catalytic one-pot scenario is also referred to as 'in-cell mediation'. [12] In cases where the potential of the mediator couple exceeds the substrate potential, a two-step protocol has to be applied, wherein the mediator is used in stoichiometric quantities and the electrochemical activation is carried out prior to addition of substrate (Scheme 1, right). Such scenarios, where the mediator fulfills the role of an electrogenerated reagent rather than that of an electrocatalyst, are often denoted as 'ex-cell mediated processes'. ${ }^{[12}$ Although this approach does not reduce the reaction overpotential and requires stoichiometric amounts of the mediator, it can be useful if it is able to induce a specific reaction mechanism and when the desired selectivity is not achieved otherwise. Both the ex-cell and the in-cell mediated approach are summarized under the term 'indirect electrosynthesis'. [12] Several examples which illustrate the concept are discussed in Sections 2.2 and 2.3.

\subsection{Homogeneous Electrocatalysis}

To illustrate the principle of homogeneous electrocatalysis, the example of electrochemical conversion of $\mathrm{CO}_{2}$ to $\mathrm{CO}$ will first be used. The activation of the relatively inert $\mathrm{CO}_{2}$ molecules by molecular electrocatalysts has received much attention during the last decade. ${ }^{[5]}$ While considerable progress has been achieved with respect to the efficiency and the mechanistic understanding, many of the reported catalysts are dependent on difficult-to-synthesize ligand structures (e.g. macrocycles or pincer ligands) and/or on costly transition metals such as Re, Pd or Ru. ${ }^{[5]}$ Consequently, the development of inexpensive, effective and robust systems still represents one of the major challenges in the field. A part of our research program is therefore devoted to the investigation of such catalytic systems. ${ }^{[16-18]}$ Due to its abundance in earth's crust, iron is a particularly interesting candidate for the metal center. However, only few iron-based systems have been reported to date for the electroreduction of $\mathrm{CO}_{2},{ }^{, 19-21]}$ whereby iron porphyrins carrying trimethylanilinium groups currently constitute the state of the art with respect to the catalytic rate. ${ }^{[22]}$ The tedious synthesis of these catalysts, however, constitutes a significant drawback. In this context, we have recently reported on the use of iron(0)-cyclopentadienones (Fe-Cat, see Scheme 2) as efficient and robust catalysts for the electrochemical conversion of $\mathrm{CO}_{2}$ to $\mathrm{CO} \cdot{ }^{[17,18]}$ An interesting aspect with regard to synthetic access is that both the cyclopentadienone ligand and the entire catalyst unit are generated in a single step from $\mathrm{Fe}_{2}(\mathrm{CO})_{9}$ and a diyne via a $[2+2+1]$ cycloaddition. ${ }^{[23,24]}$

Cyclic voltammetry represents a particularly useful technique to study the feasibility of electrocatalytic processes and to investigate the reaction mechanisms. The typical procedure is illustrated by the case of the $\mathrm{Fe}$-catalyzed $\mathrm{CO}_{2}$ electroreduction (see Fig. 1). First, Fe-Cat is examined in the absence of $\mathrm{CO}_{2}$ (under argon atmosphere, black solid line) to measure the potential of the catalyst activation, whereby an irreversible peak at approx. $-1.4 \mathrm{~V}$ and a quasi-reversible redox couple at approx. $-1.5 \mathrm{~V}$ are detected. Upon repetition of the measurement under $\mathrm{CO}_{2}$ atmosphere, a dramatic increase of the current is observed, which is caused by the turnover of $\mathrm{CO}_{2}$ by $\mathbf{F e}$-Cat ('catalytic current', red dashed line). The electrogenerated catalytically active Fe species is thus continuously reoxidized by the chemical reaction, whereby the catalytic current increases with higher rates of the homogeneous reaction. The measurement under $\mathrm{CO}_{2}$ atmosphere without catalyst (blue dotted line) shows that in the investigated potential range no conversion is possible without the catalyst.

Controlled potential electrolysis experiments revealed that useful current densities $\left(3-4.3 \mathrm{~mA} \mathrm{~cm}^{-2}\right.$ at $[$ Fe-Cat $]=0.5 \mathrm{mM}$, corresponds to a turnover frequency of 354 and $728 \mathrm{~s}^{-1}$, respectively) can be achieved while good catalyst lifetimes ( $>24 \mathrm{~h}$ ) were observed. ${ }^{[17,25]}$ With a Faradaic efficiency $(F E)$ of $96 \%$, almost exclusive selectivity for $\mathrm{CO}$ generation is maintained throughout the entire course of the electrolysis. Since in general, the electrocatalytic $\mathrm{CO}$ reduction requires addition of Lewis or Bronsted acids, one of the unusual features of the Fe-cyclopentadienone

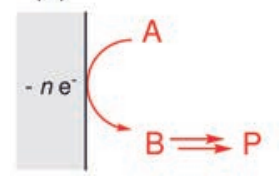

(2)

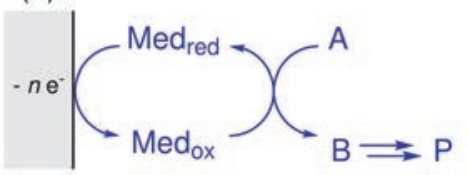

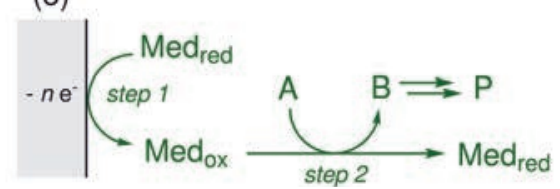

Scheme 1. Principle of direct electrolysis (case 1), homogeneous electrocatalysis ('in-cell mediation', case 2) and electrogenerated reagents ('ex-cell mediation', case 3). 


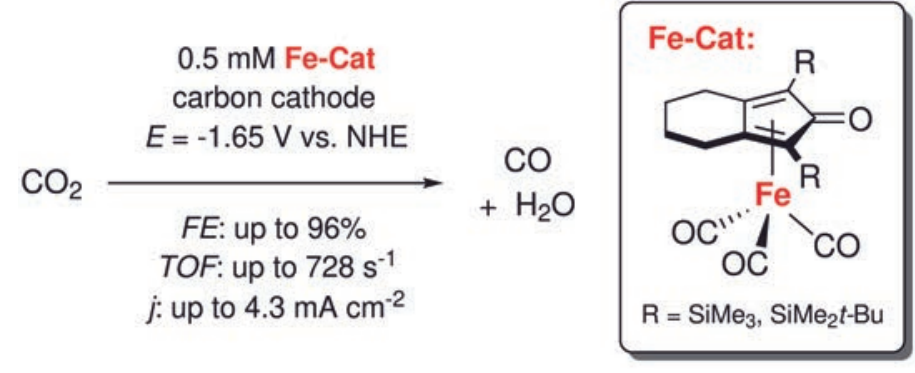

Scheme 2. Electrochemical conversion of $\mathrm{CO}_{2}$ to $\mathrm{CO}$ using $\mathrm{Fe}(0)$-cyclopentadienone catalysts.

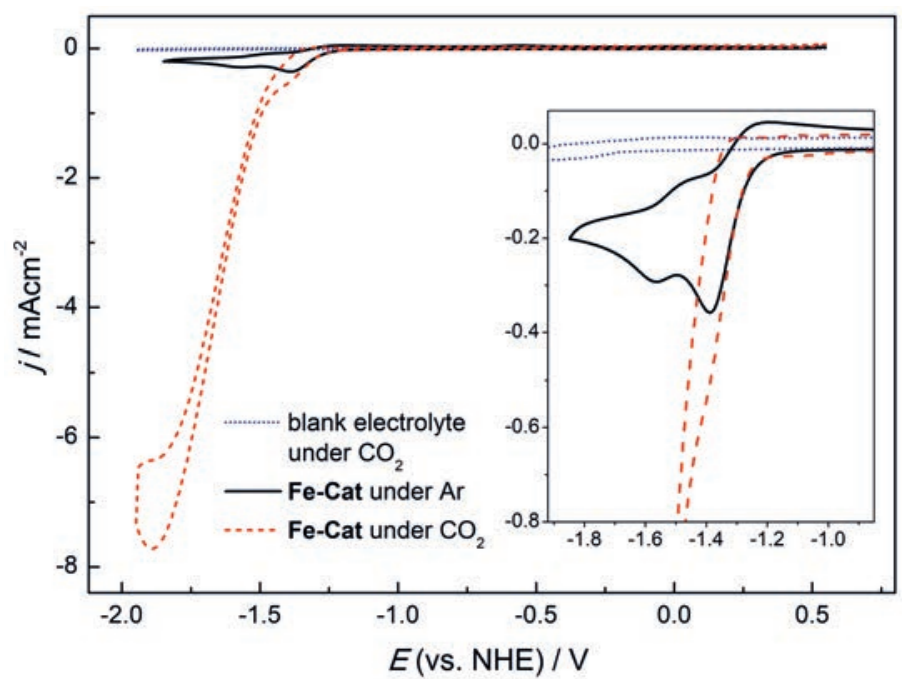

Fig. 1. Voltammetric profiles of $0.5 \mathrm{mM} \mathrm{Fe}-\mathrm{Cat}$ under Ar (solid black line) and under $\mathrm{CO}_{2}$ (dashed red line). Adapted from ref. [17] with permission from The Royal Society of Chemistry.

system is that catalysis proceeds efficiently in aprotic electrolytes in absence of acidic additives (electrolyte: $0.1 \mathrm{M} \mathrm{NBu}_{4} \mathrm{ClO}_{4}$ in dry acetonitrile). The mechanism was explored using cyclic voltammetry, kinetic simulations, DFT calculations, FTIR spectroelectrochemistry and control experiments. ${ }^{[18]}$ Several catalytic intermediates were directly detected (including a hitherto unknown $\mathrm{Fe}-\mathrm{Fe}$ dimer as active species and an $\mathrm{Fe}$ hydride as the product of catalyst deactivation).

A further case which illustrates the benefit of homogeneous electrocatalysis is represented by the electrochemical oxidation of alcohols. In numerous cases, a direct anodic conversion is inefficient or impossible due to the high potentials required for the oxidation, which particularly applies to aliphatic alcohols. [26] Furthermore, many functional groups are not compatible with the potential required for oxidation of the hydroxy group. In contrast, the electrocatalyzed conversion not only affords superior results with respect to selectivity and functional group tolerance, but can even facilitate reactions which are not possible via direct anodic oxidation. ${ }^{[27]}$ A popular catalytic system is based on $N$-oxyl compounds, in particular the TEMPO radical (see Scheme 3).

Contrary to the conventional non-electrochemical TEMPO oxidations, the electrochemical method does not require the use of stoichiometric amounts of terminal oxidant (e.g. $\mathrm{NaClO}$ ). Using TEMPO as electrocatalyst enables selective transformations of primary and secondary alcohols to the corresponding carbonyl compounds at potentials around $0.3 \mathrm{~V}$ vs. $\mathrm{Ag} / \mathrm{AgNO}_{3}$ which is between $0.8 \mathrm{~V}$ and $>1.6 \mathrm{~V}$ below the potential required for direct alcohol oxidation (depending on the substituents $\mathrm{R}^{1}$ and $\mathrm{R}^{2}$ ). ${ }^{[27]}$ The over-oxidation of primary alcohols to the carboxylic acids is generally avoided by electrolyzing in organic solvents. ${ }^{[28,29]}$ Due to the favorable kinetics of the oxidation of primary alco-

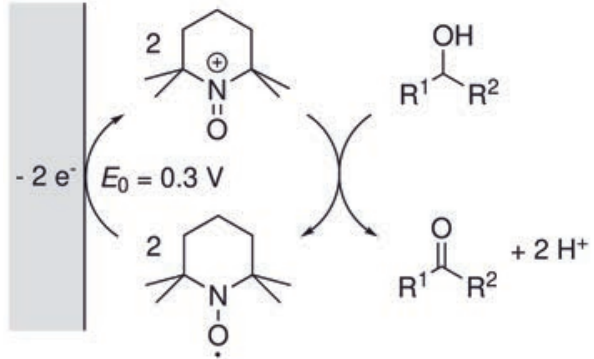

Scheme 3. Indirect anodic oxidation of alcohols using TEMPO as electrocatalyst (simplified mechanism).

hols, a selective transformation in the presence of secondary hydroxy groups is usually unproblematic. Reasonable catalytic rates are only obtained upon addition of suitable bases such as $\mathrm{K}_{2} \mathrm{CO}_{3} / \mathrm{KHCO}_{3}$ in aqueous electrolytes or 2,6-lutidine in organic media. ${ }^{29,30]}$

Since the introduction of the TEMPO mediator to electrosynthesis in the early 1980 s, ${ }^{[29]}$ numerous papers on the subject have appeared and the advances in both mechanistic understanding and synthetic applications are truly remarkable (a comprehensive review of the topic is provided in ref. [27]). A recently published contribution to the field from our laboratory is shown in Fig. 2.[31] Here we have investigated the use of soluble polymediators and polyelectrolytes in view of the simplification of separation and recycling. The idea was to use this polymer-based approach for coupling electrosynthesis with membrane-based separation processes such as ultrafiltration or dialysis. Initially we aimed at obtaining fundamental insights into the mass transport, the redox behavior and the electrocatalytic activity of the polymediators as well as gaining experience in the practical implementation of a polymediated electrolysis. Due to the robustness and reliability of the TEMPO catalyst reported before by others, ${ }^{[27]}$ we have selected the electrocatalyzed alcohol oxidation shown in Scheme 3 as model system. The polyelectrolyte HP-1 and the polymediator HP-2 (see Fig. 2, bottom) used for the study were each synthesized in two scalable steps from commercially available starting materials. ${ }^{[31]}$

Cyclic voltammetry studies carried out in $\mathrm{CH}_{3} \mathrm{CN} / \mathrm{H}_{2} \mathrm{O}$ (95:5) showed that despite the increased molecular weight and the concomitant deceleration of the diffusion of HP-2 to the electrode surface, reasonable catalytic current densities can be obtained in presence of alcohols. In fact, the slower diffusion rate is largely compensated by the accelerated kinetics of the homogeneous reaction, which is explained by an anodic shift of $E_{0}(\mathbf{H P}-2)$ with respect to $E_{0}$ (TEMPO) due to the electron-withdrawing acyloxy linker and the associated increase of the driving force. ${ }^{[32]}$ Based on the promising electroanalytical results, a protocol was developed for the preparative scale. The optimized electrolysis conditions comprise the use of a divided cell (to prevent from discharge of the active form of the polymediator on the cathode, see Fig. 3, top), an inexpensive carbon roving bundle anode, a $\mathrm{Ag} / \mathrm{AgNO}_{3}$ reference electrode (to maintain the potential of the working electrode close to $E_{0}(\mathbf{H P}-2)$ ) and a platinum counter electrode (to facilitate cathodic $\mathrm{H}_{2}$ evolution as cathodic half-reaction).

A further notable feature of the set-up is the inexpensive sizeexclusive dialysis membrane made of regenerated cellulose which was used in order to separate the half-cells from each other. Under optimized conditions, a broad range of aliphatic, allylic and benzylic alcohols was transformed into the corresponding carbonyl compounds with FEs between $58 \%$ and $97 \%\left(Q \mathrm{~mol}^{-1}=1.8 \mathrm{~F}\right)$. The reusability of HP-1 and HP-2 was demonstrated in two series of five subsequent electrolyses (see Fig. 3, bottom). The polymers were recovered after each electrolysis by ultrafiltration (red bars) and dialysis (green bars), respectively. While the recycling studies using dialysis show that $\mathbf{H P - 1}$ and $\mathbf{H P - 2}$ are suitable for at least 

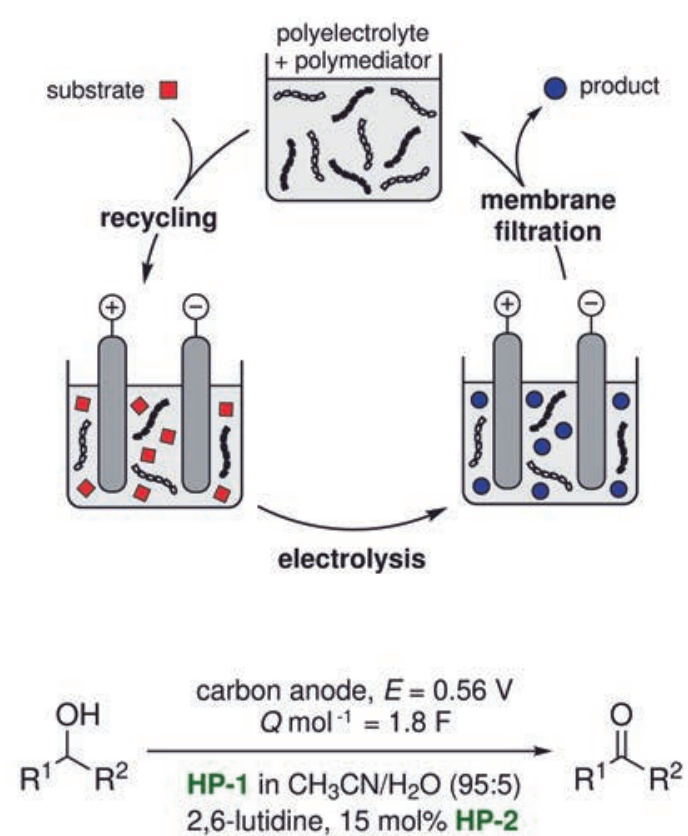

$\mathrm{R}^{1}=$ alkyl, allyl, benzyl

$\mathrm{R}^{2}=\mathrm{H}$, alkyl

FE: $58-97 \%$

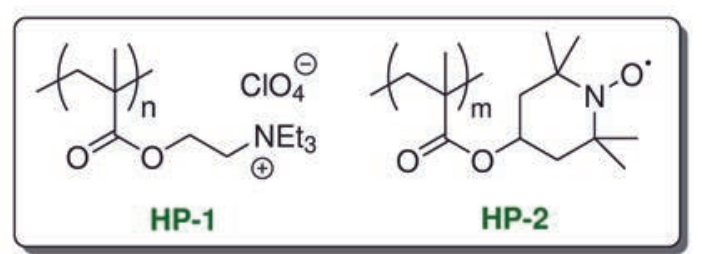

Fig. 2. Top: Electrolysis and recycling scheme. Bottom: Indirect anodic oxidation of alcohols using a polyelectrolyte (HP-1) and a polymediator (HP-2). Adapted from ref. [31] with permission from John Wiley \& Sons.

five runs without loss of activity and conductivity, the recovery by means of ultrafiltration leads to a stable performance for only three runs. The loss of activity in the fourth run can be explained by a non-quantitative retention of the polymers in the pressure-driven ultrafiltration process, which may be improved in the future by a careful adaption of the pore size distribution to the molecular weight distribution of the polymer. While the present work has already shown important prerequisites for the use of polymediators and polyelectrolytes in electrosynthesis, further studies on the influence of the molecular weight distribution and on the generalizability of the concept are underway.

\subsection{Electrogenerated Reagents}

As described in Section 2.1, mediators are sometimes used in stoichiometric quantities and are electrochemically activated before the substrate is added. This so-called ex-cell process becomes necessary when the mediator is more difficult to oxidize (or to reduce) than the starting material. Iodoarenes and the corresponding anodically generated hypervalent iodine(III) compounds are a typical example. Since iodoarenes exhibit high redox potentials (typically $>1.5 \mathrm{~V}$ vs. $\mathrm{Ag} / \mathrm{AgNO}_{3}$ ), their oxidation in presence of organic compounds carrying redox-active functional groups is usually not possible. Consequently, the iodine(I)/iodine(III) couple is preferably employed in ex-cell processes while only very few electrocatalytic applications have been reported. [33]

In principle, the electrosynthesis of three different classes of iodine(III) compounds is known (see Scheme 4). ${ }^{[34]}$ In all of the reported cases, the electrogeneration of the iodine(III) species is achieved via two electron oxidation of the iodoarene precursor $\mathbf{1}$, whereby the structure of the hypervalent species depends on the composition of the electrolyte solution. In absence of nucleo-
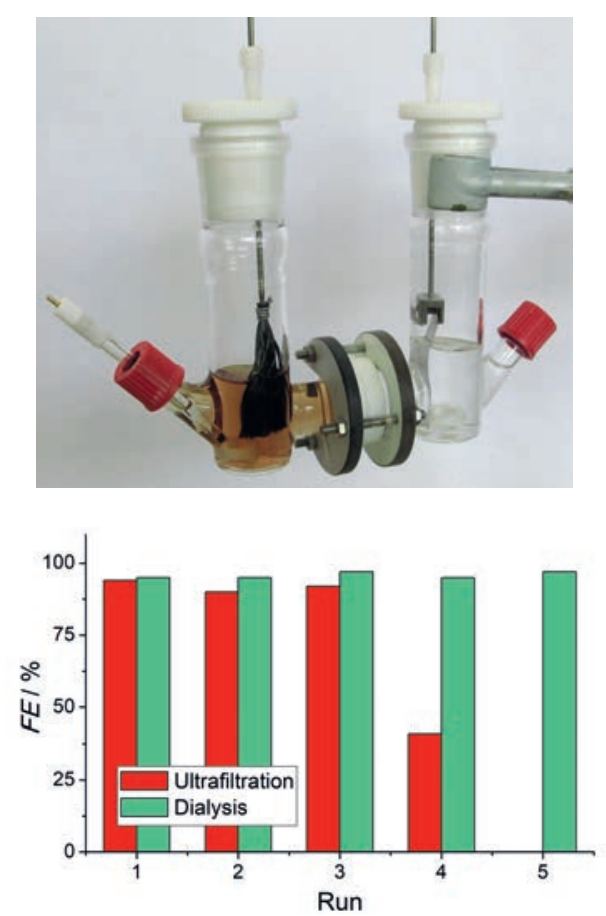

Fig. 3. Top: Divided cell used for the anodic alcohol oxidation according to Fig. 2. Bottom: Results of the recycling studies using 1-octanol as test substrate. Reprinted from ref. [31] with permission from John Wiley \& Sons.

philes, for example, the iodine(III) unit is stabilized by homocoupling via electrophilic aromatic substitution, thereby rendering diaryl iodonium compound 2. Employing an excess of a second arene reactant leads to a selective cross-coupling thus generating

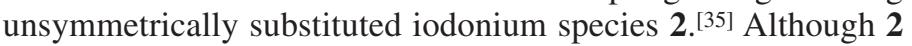
can in principle be used as reagents for arylation reactions, ${ }^{[36]}$ their application as mediators in indirect electrosynthesis has not yet been reported. In contrast, electrochemically generated (difluoroiodo)arenes (3) and (dialkoxyiodo)arenes (4) have been extensively investigated with respect to their reactivity toward different classes of organic compounds and on the basis of these results, a number of useful indirect electrosyntheses were developed. ${ }^{[37]}$ The electrogeneration of compounds 3 and $\mathbf{4}$ proceeds via two electron oxidation of $\mathbf{1}$ in $\mathrm{NEt}_{3} \cdot \mathrm{nHF}$ ionic liquids (with $3<\mathrm{n}<5$ ) and in fluorinated alcohols, respectively. In both cases, protons are reduced at the cathode, rendering $\mathrm{H}_{2}$ as the only byproduct.

Since the seminal work by Fuchigami et al. in the 1990s on the indirect anodic partial fluorinations using compounds of type 3 as a mediator, ${ }^{[38]}$ the subfield has evolved continuously. In our contributions we have focused on the hitherto poorly understood group of dialkoxy- $\lambda^{3}$-iodanes $\mathbf{4}$ and studied their electrochemistry and reactivity, while simultaneously addressing the supporting electrolyte issue described in the introduction. A further separation problem is constituted by the low polarity of the iodoarenes, which complicates the isolation of the mediator from the product mixture. In this context, we have developed and tested mediators 1a-c (see Scheme 5, bottom left), which contain ionic groups providing ionic conductivity and facilitating the workup of the reaction mixture. ${ }^{[39,40]}$

For several reasons, the anodic oxidation of $\mathbf{1 a - c}$ into the corresponding dialkoxy- $\lambda^{3}$-iodanes $\mathbf{4 a}-\mathbf{c}$ as well as the subsequent mediated reactions are preferentially carried out in 1,1,1,3,3,3-hexafluoroisopropanol (HFIP). First, the solvent exhibits an extraordinarily high anodic stability, ${ }^{[41]}$ which allows for selective oxidation of the iodoarenes without solvent degradation. Second, the resulting hypervalent iodine species $\mathbf{4 a - c}$ are stable in solution for several days. Third, HFIP is known to have a rate- 
enhancing effect on iodine(III)-mediated transformations and is therefore often employed as additive or solvent. ${ }^{[42]}$ Finally, the low $\mathrm{pK}_{\mathrm{a}}$ value of 9.3 enables $\mathrm{H}_{2}$ evolution as a benign cathodic half-reaction. ${ }^{[43]}$

Exploring the reactivity of electrogenerated species $\mathbf{4 a}$ and $4 \mathbf{b}$, we found that direct oxidative intra- and intermolecular $\mathrm{C}-\mathrm{N}$ coupling between arenes and amide groups can be induced, enabling the synthesis of substituted carbazoles $\mathbf{6}$ and amide-arene cross-coupling products 7 in a two-step one-pot protocol (see Scheme 6). ${ }^{[39,40]}$ In addition, we have shown that $4 \mathbf{a}$ enables the intramolecular cyclization of phenolic imines $\mathbf{8}$ to benzoxazoles 9 as well as of allylamide $\mathbf{1 0}$ to oxazolidine 11. ${ }^{[40,44]} \mathrm{A}$ comparison between the oxidation potentials of species $4 \mathbf{a}-\mathbf{c}(1.9-2.2$ $\mathrm{V}$ vs. $\mathrm{Ag} / \mathrm{AgNO}_{3}$ ) and the potential of the substrates (e.g. $1.6 \mathrm{~V}$ for species 5 with $\mathrm{R}^{1}=\mathrm{Ac}$ and $\mathrm{R}^{2}=\mathrm{H}$ ) highlights the necessity to apply the ex-cell procedure. ${ }^{[40]}$ In all cases, the ionic tags on 4 enabled electrolysis without supporting electrolyte additives, while the separation of $\mathbf{1 a} / \mathbf{1} \mathbf{b}$ from the crude products turned out to be remarkably straightforward (after removal of HFIP by distillation, a simple trituration of the remaining solid with non-polar solvents rendered $\mathbf{1 a} / \mathbf{1} \mathbf{b}$ with recovery rates of typically $>95 \%$ ).

With regard to the effort required to synthesize the redox-active salts, $\mathbf{1 b}$ is the species of choice, as it can be generated in only one step from commercially available precursors (in contrast, 1a must be synthesized in three steps). However, our studies on the

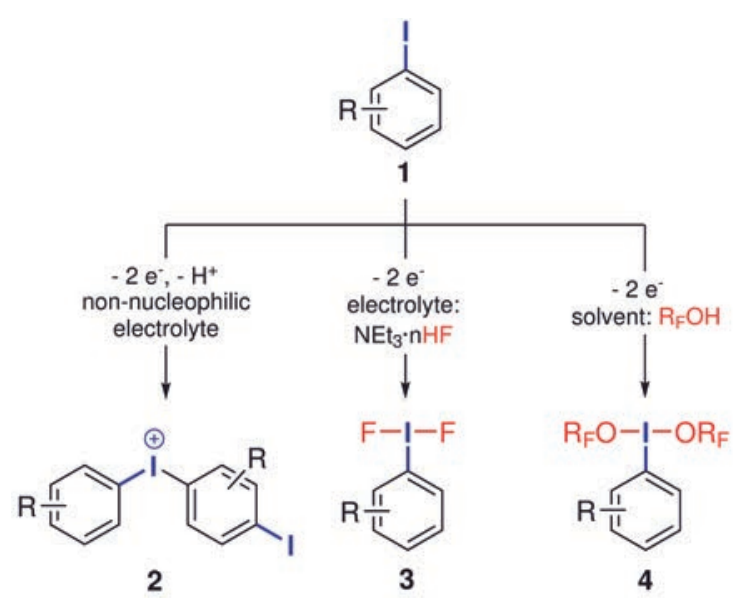

Scheme 4. Top: Anodic conversion of iodoarenes 1 into hypervalent iodine compounds 2-4.
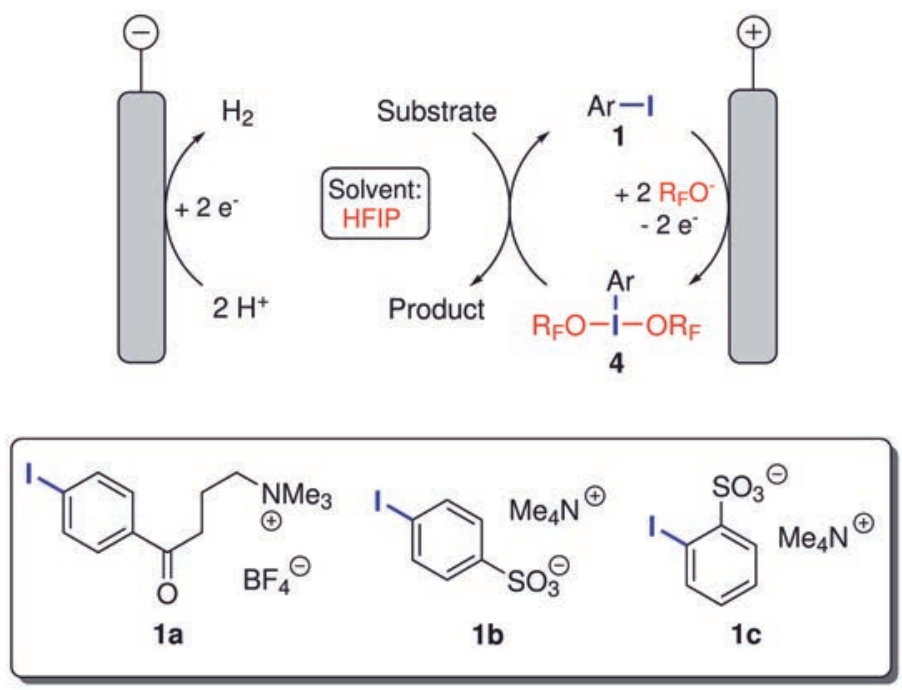

Scheme 5. Supporting electrolyte-free indirect electrosynthesis using ionically tagged iodoarenes 1a-c. applications have shown that in some instances, the anionic tag of 1b can cause problems. For example, in the oxazolidine synthesis shown in Scheme 6, the sulfonate group acted as a nucleophile, intercepting a cationic intermediate and thus inhibiting the formation of the desired product $\mathbf{1 1}$. In contrast, reagent $\mathbf{4 a}$ with its cationic tag induced the desired reaction in good selectivity. Species 1a can therefore be considered as the mediator of choice whenever the nucleophilic interception of intermediates would alter the course of the reaction.

\section{Electrochemical Catalysis and Redox-neutral Reactions}

The vast majority of catalytic reactions in electrosynthesis are based on the use of electrocatalysts to reduce the overpotential and/or to adjust the desired selectivity as described in Sections 2.1 and 2.2. A much less explored possibility to combine catalysis with electrosynthesis is the use of sub-stoichiometric amounts of charge to induce redox-neutral reactions. Instead of a catalyst facilitating an electrochemical reaction, this scenario is based on a heterogeneous electron exchange that catalyzes a chemical transformation $\mathrm{A} \rightarrow \mathrm{P}$ which in total does not require any charge transfer. ${ }^{[13]}$ Such cases are generally referred to as 'electrocatalysis' or 'electrochemical catalysis', whereby the latter term will be used here to avoid confusion with the mediated processes shown in Section 2.2.

In principle, electrochemical catalysis can be useful when a concerted redox-neutral reaction $\mathrm{A} \rightarrow \mathrm{P}$ is thermodynamically favorable but kinetically hindered (e.g. due to bond dissociation/ formation, stereoisomerization, etc.). The principle is shown in Scheme 7 using the example of an anodic hole-induced process. The initial electron exchange between the electrode and A leads to the formation of $\mathrm{A}^{-+}$(left). After spontaneous transformation into $\mathrm{P}^{\cdot+}$, the hole can be returned either to the electrode $\left(\mathrm{ECE}^{\mathrm{b}}\right.$ mechanism) or passed to another molecule A (radical chain mechanism). Aside from this stepwise mechanism, a process where the initial electron transfer and the chemical step proceed in a concerted fashion is also conceivable (right). For both scenarios, the injected holes can be regarded as catalysts, as it was previously suggested
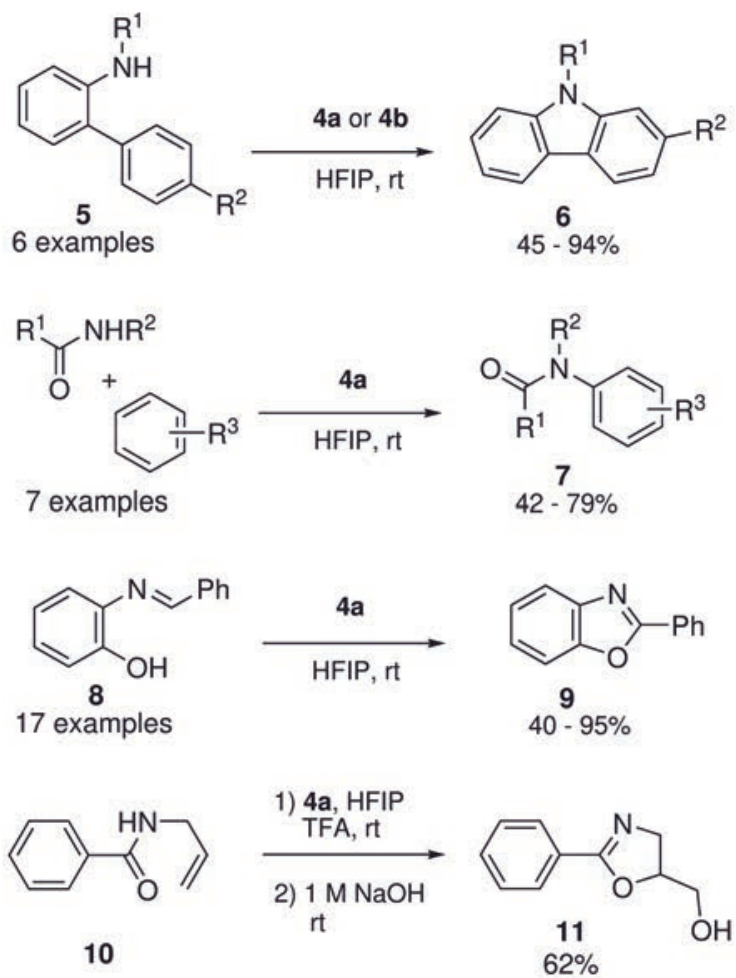

Scheme 6. Synthetic applications of electrogenerated $\lambda^{3}$-iodanes $4 a$ and 4b. 
for conventional radical and ion chain reactions. ${ }^{[45]}$ Of course, the same principle also applies to cathodically induced electron catalysis.

The Newman-Kwart rearrangement (NKR) of $O$-aryl thiocarbamates 12 to the corresponding $S$-aryl compounds 13 recently reported by us is a typical example for electrochemical catalysis. ${ }^{[46]}$ Generally, the NKR constitutes the key-step in the synthesis of thiophenols from phenols and involves the energy-rich zwitterionic transition state TS-1 (see Scheme 8). ${ }^{[47]}$ Due to the high activation barrier, temperatures between 200 and $300{ }^{\circ} \mathrm{C}$ are required, depending on the aryl substitution (electron-deficient arenes react much faster than electron-rich ones). We have shown that electrochemical catalysis, however, enables the NKR to proceed at room temperature. From the practical point of view, it is important to note that the reactions can be carried out with the simplest equipment (undivided cell, galvanostatic conditions, carbon anode). As for the electrogeneration of hypervalent iodine compounds (Section 2.3), the use of fluorinated alcohols (preferentially HFIP in combination with $\mathrm{NBu}_{4} \mathrm{ClO}_{4}$ as supporting electrolyte) turned out to be crucial for the conversion. The optimized conditions were successfully applied to the NKR of 21 different $O$-aryl thiocarbamates carrying various substituents in ortho, meta and para position, whereby the yields ranged from $32 \%$ (13h) to 95\% (13a) and the turnover number per transferred electron $(T O N)$ from 0.3 (13e) to 7.2 (13f). To demonstrate the practicality for more complex scaffolds, the $O$-aryl thiocarbamate of estradiol (an estrogen steroid hormone) was successfully converted into the corresponding $S$-aryl derivative 13i. The scope is limited by electron-deficient substrates (e.g. with halo or nitro substituents in ortho and/or para position). In these cases, no conversion was observed upon application of the standard electrolysis conditions. The reactivity for the electrochemical reaction is thus complementary to the thermally induced version, where electron-deficient arenes react much faster than electron-rich ones. These and other observations led to formulation of the mechanism depicted in Scheme 6. Here, electrogenerated $\mathbf{1 2}^{\mathbf{*}}$ rearranges to $\mathbf{1 3}^{\circ+}$ via the spirocyclic transition state TS-2, followed by reduction to $\mathbf{1 3}$ either via an $\mathrm{ECE}^{\mathrm{b}}$ pathway and/or via a radical chain process.

While the low reaction temperature and the complementary reactivity with respect to the thermal NKR constitute attractive features, the necessity for a supporting electrolyte additive is not ideal. For example, $3.4 \mathrm{~g}$ of $\mathrm{NBu}_{4} \mathrm{ClO}_{4}$ are required for the conversion of $2.1 \mathrm{~g}$ of $12 \mathrm{a}$ under optimized batch conditions. Therefore, we were interested in lowering the supporting electrolyte load and decided to test the reaction in a microflow reactor. Compared to typical batch cells, a decrease of the required salt concentration and an improved mass transport can be expected when a microflow cell with a parallel plate design is employed. ${ }^{[48,49]}$ For our studies we used a simple custom-made sandwich-type cell consisting of a glassy carbon working electrode and a platinum counter electrode, which are separated from each other by a PTFE
(1)

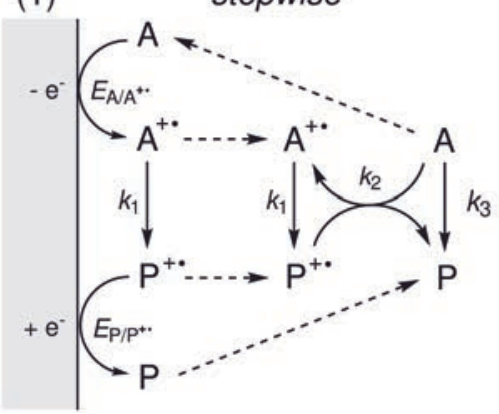

(2) concerted

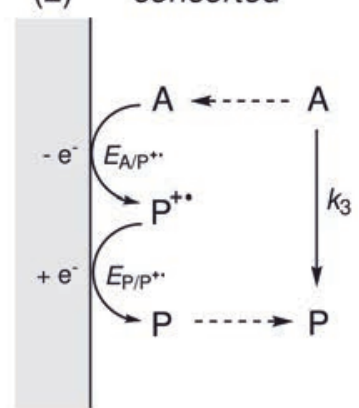

Scheme 7. Principle of electrochemical catalysis using the example of a hole-induced transformation. Adapted from ref. [13] with permission from John Wiley \& Sons. sheet with a rectangular notch $(3.0 \times 0.3 \mathrm{~cm})$ that defines the reaction channel (see Fig. 4).

Interestingly, we found that with interelectrode gaps up to 0.5 $\mathrm{mm}$, the NKR proceeds efficiently without supporting electrolyte additive. Under these conditions, the anodically generated cation radicals seem to render sufficient conductivity for closing the electric circuit. Under optimized flow conditions, the conversion of 12a led to quantitative yields in $\mathbf{1 3} \mathbf{a}$ with a product forming rate $(P F R)$ of $0.89 \mathrm{~g} \mathrm{~h}^{-1}$, a turnover number per transferred electron $\left(T O N_{\mathrm{e}}\right)$ of 6.3 , a flow rate $(\dot{V})$ of $1.0 \mathrm{~mL} \mathrm{~min}^{-1}$ and a current density (j) of $19.9 \mathrm{~mA} \mathrm{~cm}^{-2}$ (see Table 1, entry 1). It is worth noting that after removal of the solvent by distillation, the product was obtained in analytical purity without further purification. Additional experiments showed that the PFR can be further improved by increasing the feed concentration and the current density, albeit with a decrease in conversion and $T O N_{\mathrm{e}}$ (entries 2 and 3).

\section{Conclusion}

Although electrosynthesis is one of the more practical and sustainable preparative methods, the final breakthrough of the technology with respect to the conversion of carbon-based compounds still faces major challenges. Three of these challenges have been discussed in this review using examples from our laboratory. The
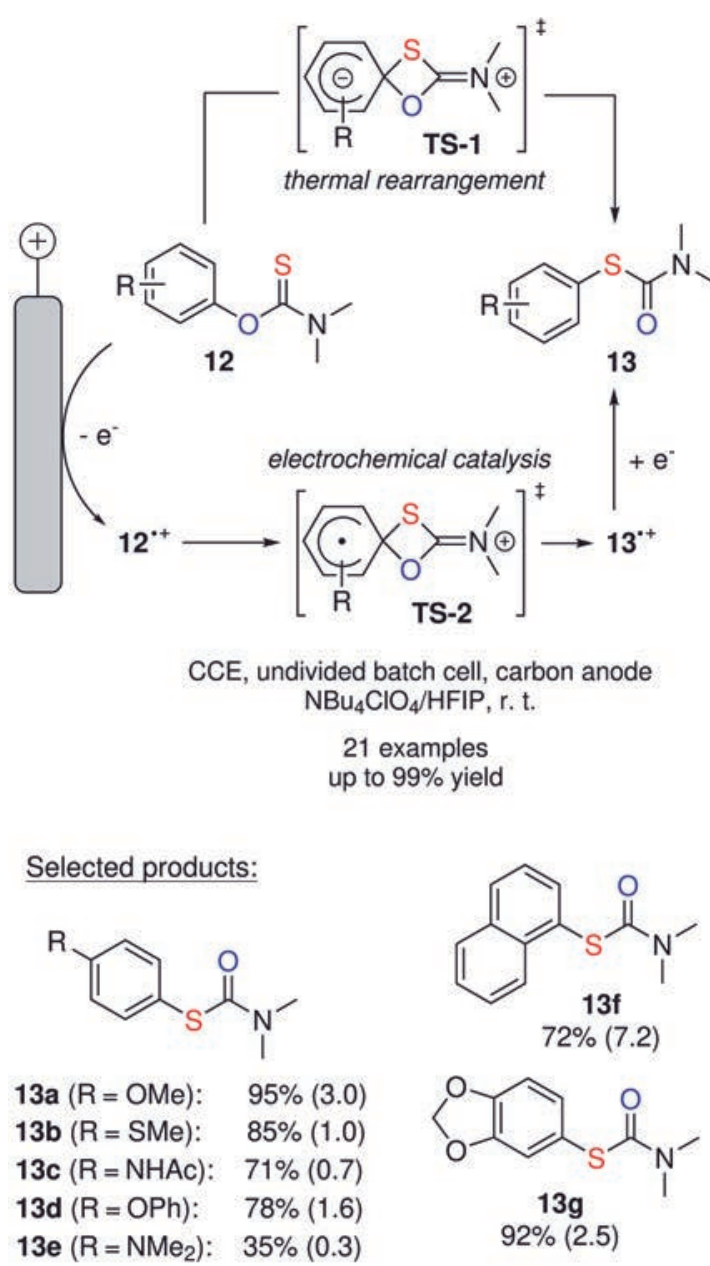

$72 \%(7.2)$
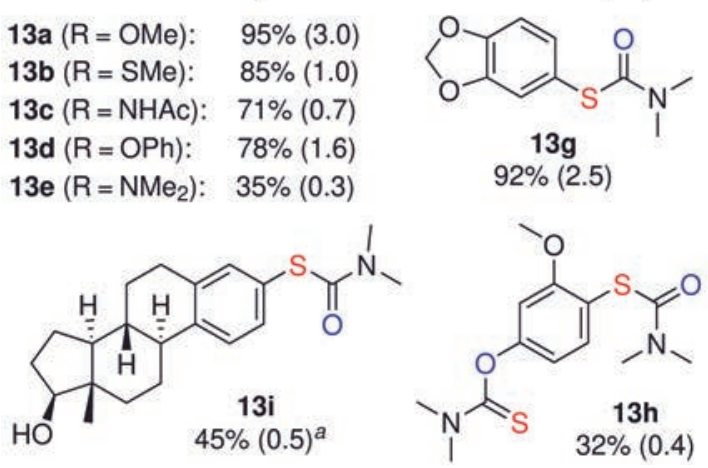

Scheme 8. Electrochemically catalyzed Newman-Kwart rearrangement and selected products. Isolated yields with $T O N_{e}$ in parenthesis. ${ }^{a}$ Electrolysis under reflux conditions (bp of HFIP: $58^{\circ} \mathrm{C}$ ). 
first issue is associated with the kinetic inhibition of the electron transfer between electrode and substrate, which increases the energy consumption (overpotential) and often leads to a decrease in selectivity. By the examples of the $\mathrm{CO}_{2}$ reduction catalyzed by iron cyclopentadienones and the TEMPO-mediated alcohol oxidation (Section 2.2), homogeneous electrocatalysis was presented as a way to overcome these issues.

The second challenge is the apparent restriction of electrosynthesis to redox processes. Using the example of the NewmanKwart rearrangement, it was demonstrated how a redox-neutral process can be facilitated by electrolysis (Section 3). The underlying principle is referred to as electrochemical catalysis and has the potential to massively expand the scope of electrosynthesis in the future.

The third challenge in the focus of this article is associated with the use of large amounts of supporting electrolyte additives. While the use of a microflow reactor as described in Section 3 is a common approach to decrease the required supporting electrolyte load, two further strategies were developed in our laboratory. The first approach is based on the attachment of catalyst units and ion pairs to soluble polymers (polymediators and polyelectrolytes, see Section 2.2), which allows for the separation of both components in a single step using a membrane process. In the second approach the mediator and the supporting electrolyte are merged into a single molecularly defined species. A series of investigations on ionically tagged iodobenzenes showed that under certain conditions, electrolysis in the batch reactor is possible without salt additives and that the separation and reuse of the mediator is significantly facilitated (see Section 2.3).

\section{Acknowledgements}

Financial support through a Liebig Fellowship (Fonds der Chemischen Industrie) is gratefully acknowledged.

Received: November 5, 2019
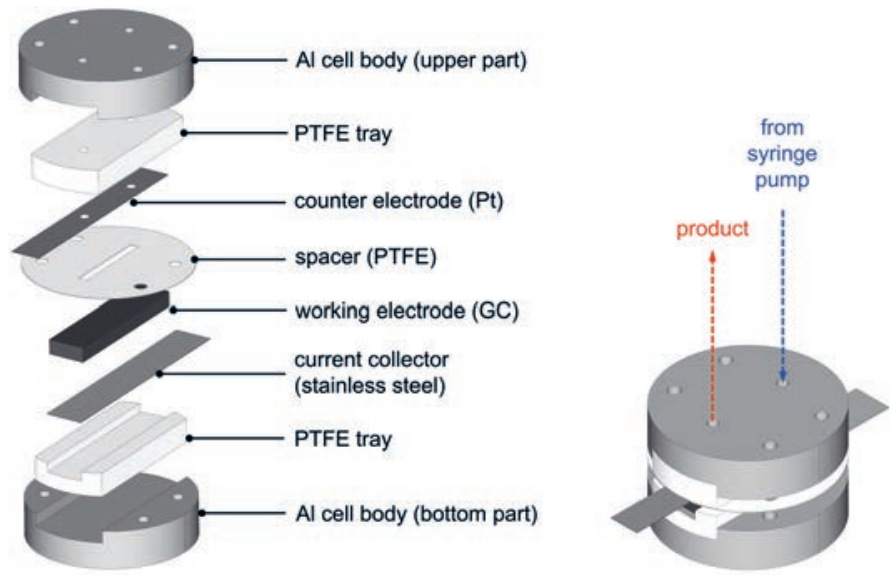

Fig. 4. Schematic illustration of the microflow cell used for the electrochemical NKR. Adapted with permission from ref. [46]. Copyright 2018 American Chemical Society.
[1] B. A. Frontana-Uribe, R. D. Little, J. G. Ibanez, A. Palma, R. VasquezMedrano, Green Chem. 2010, 12, 2099.

[2] S. R. Waldvogel, A. Wiebe, T. Gieshoff, S. Möhle, E. Rodrigo, M. Zirbes, Angew. Chem. Int. Ed. 2018, 57, 5594.

[3] E. J. Horn, B. R. Rosen, P. S. Baran, ACS Cent. Sci. 2016, 2, 302.

[4] A. Shatskiy, H. Lundberg, M. D. Kärkäs, ChemElectroChem 2019, 6, 4067.

[5] R. Francke, B. Schille, M. Roemelt, Chem. Rev. 2018, 118, 4631.

[6] V. M. Schmidt, 'Elektrochemische Verfahrenstechnik', Wiley VCH, Weinheim, 2003.

[7] An overview of the few existing industrial applications of organic electrosynthesis is provided in ref. [8] and ref. [9]. Perspectives on the feasibility of the electroreduction of $\mathrm{CO}_{2}$ on the industrial scale are given in refs. [10] and [11].

[8] H. Pütter, in: 'Organic Electrochemistry (Fourth Edition)', Ed. H. Lund, O. Hammerich, Marcel Dekker, New York, 2001, p. 1259.

[9] N. Aust, Chem. Ing. Tech. 2016, 88, 1253.

[10] D. Pletcher, Electrochem. Commun. 2015, 61, 97.

[11] J. Durst, A. Rudnev, A. Dutta, Y. Fu, J. Herranz, V. Kaliginedi, A. Kuzume, A. A. Permyakova, Y. Paratcha, P. Broekmann, T. J. Schmidt, Chimia 2015 69, 769 .

[12] R. Francke, R. D. Little, Chem. Soc. Rev. 2014, 43, 2492.

[13] R. Francke, D. Little, ChemElectroChem 2019, 6, 4373.

[14] A. Dutta, F. Bizzotto, J. Quinson, A. Zana, C. E. Morstein, M. A. Rahaman, A. C. López, M. Arenz, P. Broekmann, Chimia 2019, 73, 707.

[15] Instead of lowering the activation barrier via bonding interactions, the catalyst can also merely act as an electron shuttle between electrode and substrate in a process that is referred to as an 'outer-sphere' electron transfer. For more details see ref. [12].

[16] C. Steinlechner, A. F. Roesel, E. Oberem, A. Päpcke, N. Rockstroh, F. Gloaguen, S. Lochbrunner, R. Ludwig, A. Spannenberg, H. Junge, R. Francke, M. Beller, ACS Catal. 2019, 9, 2091.

[17] A. Rosas-Hernández, H. Junge, M. Beller, M. Roemelt, R. Francke, Catal. Sci. Technol. 2017, 7, 459.

[18] E. Oberem, A. F. Roesel, A. Rosas-Hernández, T. Kull, S. Fischer, A Spannenberg, H. Junge, M. Beller, R. Ludwig, M. Roemelt, R. Francke, Organometallics 2019, 38, 1236.

[19] N. D. Loewen, T. V. Neelakantan, L. A. Berben, Acc. Chem. Res. 2017, 50, 2362.

[20] I. Bhugun, D. Lexa, J.-M. Savéant, J. Am. Chem. Soc. 1994, 116, 5015.

[21] C. Costentin, S. Drouet, M. Robert, J.-M. Savéant, Science 2012, 338, 90.

[22] I. Azcarate, C. Costentin, M. Robert, J.-M. Saveant, J. Am. Chem. Soc. 2016, $138,16639$.

[23] H.-J. Knölker, J. Heber, C. H. Mahler, Synlett 1992, 1002.

[24] H.-J. Knölker, E. Baum, H. Goesmann, R. Klauss, Angew. Chem. Int. Ed. 1999, 38, 2064.

[25] It should be noted that while the performance of Fe-Cat is competitive compared to other homogeneous systems, it is not yet sufficient for a technical process (in particular with respect to the long-term stability). In addition to an optimization of the catalyst structure and the reaction conditions, immobilization of Fe-Cat on the electrode surface may represent a useful approach to improve the catalyst lifetime.

[26] R. Francke, T. Quell, A. Wiebe, S. R. Waldvogel, in 'Organic Electrochemistry', Ed. B. Speiser, O. Hammerich, CRC Press Taylor \& Francis Group, Boca Raton, 2016, p. 981.

[27] J. E. Nutting, M. Rafiee, S. S. Stahl, Chem. Rev. 2018, 118, 4834.

[28] J. T. Hill-Cousins, J. Kuleshova, R. A. Green, P. R. Birkin, D. Pletcher, T. J. Underwood, S. G. Leach, R. C. D. Brown, ChemSusChem 2012, 5, 326.

[29] M. F. Semmelhack, C. S. Chou, D. A. Cortes, J. Am. Chem. Soc. 1983, 105, 4492.

[30] K. Schnatbaum, H. J. Schäfer, Synthesis 1999, 1999, 864.

[31] B. Schille, N. O. Giltzau, R. Francke, Angew. Chem. Int. Ed. 2018, 57, 422.

[32] M. Rafiee, K. C. Miles, S. S. Stahl, J. Am. Chem. Soc. 2015, 137, 14751

[33] R. Francke, Curr. Opin. Electrochem. 2019, 15, 83.

[34] R. Francke, T. Broese, A. F. Roesel, in 'Hypervalent Halogen Compounds', Ed. I. Marek, John Wiley \& Sons, Hoboken, 2018, DOI: 10.1002/9780470682531.pat0960.

Table 1. Electrochemical NKR of 12a under optimized microflow conditions: Optimized product yield, TON with the corresponding process parameters. Adapted with permission from ref. [46]. Copyright 2018 American Chemical Society.

\begin{tabular}{|c|c|c|c|c|c|c|c|}
\hline Entry & $c_{\text {feed }}[\mathbf{m M}]$ & $\dot{V}\left[\mathbf{m L ~} \mathbf{m i n}^{-1}\right]$ & $j\left[\mathrm{~mA} \mathrm{~cm}^{-2}\right]$ & $12 \mathbf{a}[\%]^{b}$ & $13 \mathbf{a}[\%]^{b}$ & $T O N_{\mathrm{e}}$ & $P F R\left[\mathrm{~g} \mathrm{~h}^{-1}\right]$ \\
\hline $1^{c}$ & 70 & 1.0 & 19.9 & $<1$ & $>99$ & 6.3 & 0.89 \\
\hline $2^{d}$ & 100 & 1.1 & 31.3 & 2 & 98 & 6.2 & 1.37 \\
\hline $3^{d}$ & 150 & 1.2 & 51.1 & 11 & 89 & 5.6 & 2.03 \\
\hline
\end{tabular}

${ }^{a}$ Conditions: no supporting electrolyte, r. t., single pass, undivided cell, $Q \mathrm{~mol}^{-1}=0.16 \mathrm{~F}, \mathrm{WE}=$ glassy carbon, $\mathrm{CE}=\mathrm{Pt}, A$ $=0.9 \mathrm{~cm}^{2}$, interelectrode distance: $110 \mu \mathrm{m}$. ${ }^{b}$ Isolated yields. ${ }^{c}$ Batch size: $2.0 \mathrm{~g} .{ }^{d}$ Batch size: $1.0 \mathrm{~g}$. 
[35] K. Watts, W. Gattrell, T. Wirth, Beilstein J. Org. Chem. 2011, 7, 1108.

[36] P. Villo, B. Olofsson, in 'Hypervalent Halogen Compounds', Ed. I. Marek, John Wiley \& Sons, Hoboken, 2018, DOI: 10.1002/9780470682531. pat0950.

[37] M. Elsherbini, T. Wirth, Chem. Eur. J. 2018, 24, 13399.

[38] T. Fuchigami, T. Fujita, J. Org. Chem. 1994, 59, 7190.

[39] T. Broese, R. Francke, Org. Lett. 2016, 18, 5896.

[40] A. F. Roesel, T. Broese, M. Májek, R. Francke, ChemElectroChem 2019, 6, 4229-4237.

[41] R. Francke, D. Cericola, R. Kötz, D. Weingarth, S. R. Waldvogel, Electrochim. Acta 2012, 62, 372.

[42] A. Yoshimura, V. V. Zhdankin, Chem. Rev. 2016, 116, 3328.

[43] I. Colomer, A. E. R. Chamberlain, M. B. Haughey, T. J. Donohoe, Nat. Rev. Chem. 2017, 1, 0088 .

[44] O. Koleda, T. Broese, J. Noetzel, M. Roemelt, E. Suna, R. Francke, J. Org. Chem. 2017, 82, 11669

[45] A. Studer, D. P. Curran, Nat. Chem. 2014, 6, 765.

[46] T. Broese, A. F. Roesel, A. Prudlik, R. Francke, Org. Lett. 2018, 20, 7483.

[47] G. C. Lloyd-Jones, J. Moseley, J. Renny, Synthesis 2008, 661.

[48] K. Watts, A. Baker, T. Wirth, J. Flow Chem. 2015, 4, 2.

[49] M. Atobe, H. Tateno, Y. Matsumura, Chem. Rev. 2017, 118, 4541

\section{License and Terms}

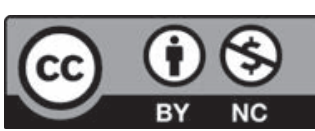

This is an Open Access article under the terms of the Creative Commons Attribution License CC BY_NC 4.0. The material may not be used for commercial purposes.

The license is subject to the CHIMIA terms and conditions: (http:// chimia.ch/component/sppagebuilder/?view=page $\& i d=12$ ).

The definitive version of this article is the electronic one that can be found at doi:10.2533/chimia.2020.49. 